\title{
Tuberculosis in Asians
}

\author{
J. A. INNES \\ B.Sc., M.B., Ch.B., F.R.C.P. (Edin.) \\ Department of Communicable and Tropical Diseases, East Birmingham Hospital, Bordesley Green East, \\ Birmingham B9 5ST
}

\begin{abstract}
Summary
The incidence of tuberculosis among Asian immigrants in the United Kingdom greatly exceeds that in the white population. There are differences in the ageand sex-distribution of cases and in the frequency of of the disease at various sites, non-respiratory disease being disproportionately common. These differences are described and the need for more vigorous application of case finding and preventive measures is discussed.
\end{abstract}

\section{Introduction}

Tuberculosis in the Asian immigrant population of the United Kingdom displays striking differences from the disease as it is seen in the indigenous white population. These differences, which are both quantitative and qualitative, are very much easier to describe than to explain.

\section{Quantitative differences}

Between 1963 and 1979 the annual number of notifications of tuberculosis in England and Wales declined steadily from 18500 to 10500 cases. This overall trend conceals 2 contrasting epidemiological patterns: among the white population the decline was more rapid with a fall of $60 \%$ in annual notifications in the Birmingham Metropolitan District over this period, whereas among the immigrant population (largely from the Indian sub-continent) the annual notification rate rose. However, the increasing proportion of Asian cases in the overall notification figures in England and Wales is not the result of the falling rate in the white population and the increasing size of the immigrant population alone. Within the Asian immigrant group the annual incidence of tuberculosis per head of population is very much greater than that in the white population. This effect was most recently measured in a national notification survey in 1978-1979 (M.R.C. Tuberculosis and Chest Diseases Unit, 1980) making use of population data from the National Dwelling and Housing Survey. In that study, the annual notification rate of tuberculosis in Indian, Pakistani and Bangladeshi immigrants $(354 / 100000)$ exceeded the rate in the white population by 38 times. As in the white population, the rates were highest in the oldest age group $(>55$ years) but, unlike the white population, the rate for females exceeded that for males in all age groups.

The annual incidence of tuberculosis in British born children of Asian immigrant parents $(63 / 100000)$ is less than that in Asian immigrant children $(114 / 100000)$ but very much higher than that in white children $(3 / 100000)$. The high incidence in this group is presumably related to the frequency of tuberculosis in Asian adults with whom they live, their domestic overcrowding and possibly to the fact that such children often visit the Indian subcontinent for periods of months or years. In many parts of the United Kingdom neonatal B.C.G. immunization is offered to children born to Asian parents. The protective effect of this practice is uncertain.

\section{Qualitative differences}

Qualitative differences between tuberculosis (TB) in the white and Asian population, can be identified at several different levels. Of all white cases $80 \%$ are of respiratory disease, $16 \%$ are of non-respiratory disease and $4 \%$ are of both. By contrast, only $56 \%$ of Asian immigrant cases are of respiratory disease, $33 \%$ are of non-respiratory disease and $11 \%$ are of both. Non-respiratory disease is, therefore, much more common in Asians, as is disease at more than one anatomical site.

\section{Respiratory tuberculosis}

In the white population respiratory tuberculosis is most often seen in middle-aged and elderly men. The radiographic appearances are usually of irregular upper lobe consolidation which is often bilateral and is frequently associated with cavitation. Among Asian immigrants respiratory TB is more varied and cases are more evenly distributed by age (this is partly accounted for by the lower average age of the Asian immigant population as a whole). There is also a higher incidence of TB in Asian women than in men, whereas among the white population, male cases outnumber female cases by almost 2 to one. The female preponderance among Asian cases may reflect their more recent arrival in this country as the risk of tuberculosis is greatest in the first 2 years after arrival and declines thereafter.

In about $20 \%$ of Asian immigrant respiratory 
cases, enlargement of hilar or mediastinal lymph nodes is the only radiographic abnormality. Such patients often present with fever rather than with respiratory symptoms. Where pulmonary parenchymal involvement is present, the radiographic extent of the lesions and the incidence of cavitation is generally less than that seen in the white population. Furthermore, atypical radiographic presentations with, for example, middle or lower lobe consolidation, are more common in Asians.

As might be expected from the lesser radiographic severity the rate of bacteriological confirmation of tuberculosis by either smear or culture examination of the sputum is lower in Asian patients.

\section{Non-respiratory tuberculosis}

Non-respiratory tuberculosis is particularly common in Asian immigrants, the annual incidence per head of population being about 80 times that of the white population. Furthermore, the distribution of cases by site of disease differs significantly from that found in whites. In the white population, superficial lymph node and genito-urinary infections each account for about $30 \%$ of cases. By contrast, $56 \%$ of Asian immigrant non-respiratory cases are of lymph node disease whereas only $4 \%$ involve the genito-urinary tract. The high rate of lymph node disease in Asians is difficult to explain and it so overshadows the rate of genito-urinary disease that the impression has arisen that genito-urinary TB is rare in Asians. However, the incidence of this form of disease remains 10 times higher among Asian immigrants than it is in the white population.

The proportion of patients with non-respiratory disease at sites other than lymph nodes or genitourinary tract is similar in both ethnic groups, bone and joint disease being the next most common variety.

Not only are there differences in the frequency and anatomical sites of non-respiratory disease in Asian immigrants, but within certain sites the features of tuberculous infection may be different. The best example of this phenomenon is spinal TB which in white cases almost invariably starts at one of the anterior angles of the vertebral body spreading posteriorly to destroy the body and often involving the adjacent surface of the neighbouring vertebra. Anterior collapse and angulation of the spine may follow. In Asian immigrants, up to $40 \%$ of spinal TB presents with destructive lesions of the posterior part of the vertebral body or of elements of the neural arch (Taor, 1977), an appearance which may be confused with metastatic malignant disease.

In all ethnic groups, it is usually possible at the time of presentation with TB to define a focus of infection by clinical examination or by conventional microbiological or radiological techniques. However, among Asian immigrants it is not uncommon for TB to present with constitutional disturbances only̌ and with no easily identified focus of infection. In: such patients, liver biopsy often shows granulom formation, although usually without caseation. In $\Phi$ personal series of 21 such cases collected over $3 \Phi$ months, 13 have gone on to show overt evidence of disease during the first few months of treatment邑 The most common sites of these late manifestationg were the cervical lymph nodes.

\section{Microbiological differences}

The different clinical behaviour of TB amon: $\frac{\omega}{8}$ Asian immigrants has led to speculation that there may be differences between the organism prevalen $\bar{E}$. in the white population and that found in Asiansir Microbiological differences do exist but they do not account for the observed clinical differences. Drug resistance, particularly to streptomycin and isoniazid, is more common in Asian immigrants. Thiङ is almost cetainly an acquired characteristic of the organism resulting from the use of inadequatetreatment regimes in Asia. More fundamentally there are differences in the distribution of myco bacteriophage types among the organisms isolate $\bar{\Phi}$ from Asian and from white patients (Grange et gl. 1977) but so far it has not been possible to demonstrate clinical consequences of this variation.

\section{Control measures}

The high incidence of TB in Asian immigrant requires the application of more vigorous contro measures. Opportunities for active case finding b chest radiography arise at the point of entry of new immigrants to the country and in the areas wher $\overrightarrow{\vec{E}}$ they first settle. Radiographic screening will not, of course, detect non-respiratory cases of TB but does have the advantage that it will detect all those. cases which are infectious to others. Immigrand children can be examined by tuberculin testing. before school entry. Those who have negative reactions can be immunized with B.C.G. while positive reactions can be examined further for active disease. The use of neo-natal B.C.G. immunization in British born children of Asian immigrants has already been mentioned.

\section{References}

GRANGE, JM., ABER, V.R., ALLEN, BW MITCHISON, D A Mikhall, J.R., McSwigGan, D.A. \& Collins, C.H. (1977 Comparison of strains of mycobacterium tuberculosis frome British, Ugandan and Asian immigrant patients. Tubercle 58, 207.

M.R.C. Tuberculosis AND Chest Diseases Unit (198 National survey of tuberculosis notifications in Englan $\$$ and Wales, 1978-1979. British Medical Journal, 281, 895?

TAOR, W.S. (1977) Bone and joint tuberculosis in Brent.o In: Abstracts of a Symposium on Extra-pulmonary Tubero culosis. Ciba Laboratories, Horsham. pp. 1-3. 